\title{
Análisis de las características geométricas del ancho de carril e infraestructura en las paradas de bus del transporte público urbano en la ciudad de Riobamba
}

Analysis of the geometric characteristics of the lane width and infrastructure in the bus stops of urban public transport in the city of Riobamba

Geoconda Marisela Velasco Castelo. ${ }^{1}$, Alexandra Patricia Guerrero Godoy. ${ }^{2}$ \& Gustavo Javier Aguilar Miranda. ${ }^{3}$

Recibido: 23-05-2020 /Aceptado: 27-06-2020 / Publicado: 03-07-2020

\begin{abstract}
.
DOI: https://doi.org/10.33262/concienciadigital.v3i3.1325

The following article focuses on the analysis of the geometric characteristics of the lane width and the infrastructure of bus stops of the urban public transport of the city of Riobamba, for which an on-site survey of the cadastre-type information has been carried out, preparing files where information such as the type was identified, identifying its geometry and the state of the stop infrastructure, as well as registering the existence of the type of horizontal and vertical signaling. The importance of bus stops lies in the comfort of users when moving around in public transport, where they are exposed to a series of inconveniences that are aggravated by the varying weather situation in our environment. There are 16 public transport lines, which are grouped into 10 groups, relating the route of the route that the transport units carry out. The investigation revealed the real and constant problems that citizens have when using public transport stops to move from a point origin to a destination. The results obtained based on the analysis reflect the existence of horizontal and vertical signaling at the stops, around $30 \%$ have the appropriate stop infrastructure, in the geometric analysis verifying the measurements of the lane width dimension 8 groups comply with the regular regulations that allow the adequate circulation of public transport units.
\end{abstract}

\footnotetext{
${ }^{1}$ Escuela Superior Politécnica de Chimborazo, Magíster en Gestión Industrial y Sistemas Productivos, Riobamba, Ecuador, geoconda.velasco@espoch.edu.ec

${ }^{2}$ Escuela Superior Politécnica de Chimborazo, Magíster en Ingeniería Vial, Riobamba, Ecuador, alexandra.guerrero@espoch.edu.ec

${ }^{3}$ Escuela Superior Politécnica de Chimborazo, Magíster en Transporte y Logística, gustavo.aguilar@espoch.edu.ec
} 
Keywords: Geometric analysis, infrastructure, urban transport, stop, signaling.

\section{Resumen.}

El siguiente artículo se enfoca en el análisis de las características geométricas del ancho del carril y de la infraestructura de paradas de bus del transporte público urbano de la ciudad de Riobamba, para lo cual se ha realizado un levantamiento in situ de la información tipo catastro, elaborando fichas donde se registró información como el tipo identificando su geometría y estado de la infraestructura de parada además el registro de la existencia del tipo de señalización horizontal y vertical. La importancia de los paraderos de bus recae en la comodidad de los usuarios al movilizarse en el transporte público, donde los mismos están expuestos a una serie de inconvenientes que se agravan con la situación climatológica variante en nuestro medio. Existen 16 líneas de transporte público las mismas que están agrupadas en 10 grupos relacionando la ruta del recorrido que realizan las unidades de transporte, en la investigación se evidenció los problemas reales y constantes que tiene la ciudadanía cuando utilizan las paradas de transporte público para trasladarse de un punto origen a un destino. Los resultados obtenidos en base al análisis reflejan la existencia de señalización horizontal y vertical en las paradas, alrededor del $30 \%$ se cuenta con la infraestructura de parada adecuada, en el análisis geométrico verificando las medidas de la dimensión del ancho de carril 8 grupos cumplen con la normativa regular que permite la circulación adecuada de las unidades de transporte público.

Palabras clave: Análisis geométrico, infraestructura, transporte urbano, parada, señalización.

\section{Introducción.}

En la actualidad a nivel de Latinoamérica estudios y proyectos de ingeniería se han convertido en estudios y proyectos desarrollados a partir de la identificación de problemas de seguridad y/o de fluidez en la red vial. La identificación de estos problemas puede ocurrir por la observación de los propios técnicos del órgano municipal, quienes están presentes cada día en las vías de la ciudad para los estudios de capacidad y de calidad de servicio, los cuales deben ser realizados permanentemente, o también a partir de las quejas o sugerencias de los ciudadanos. (Martinez Filho, A., Vasconcellos, E. A., \& Paulino, H. 2014).

Los tipos de estudios pueden ser subdivididos de acuerdo con el área que abarca o con la motivación del estudio. Según los criterios establecidos en cada órgano de tránsito estos puntos críticos pueden ser desarrollados en un punto localizado (intersección o fragmento de vía) para resolver un problema de fluidez y/o de seguridad. Análisis de carriles a lo largo de un carril vial, también para solucionar algún problema de fluidez o de seguridad, o, inclusive, para la introducción de algún nuevo sistema de prioridad del transporte público, por ejemplo, un nuevo carril para autobuses. (Martinez Filho, A., Vasconcellos, E. A., \& Paulino, H. 2014). 


\section{Fundamentación teórica.}

\section{Definición de parada de autobús.}

Se define como parada de bus o paradero en su forma más simple a la infraestructura física construida con el fin de acoger a pasajeros en la espera de un transporte público, es el mobiliario público que resguarda a los usuarios de la intemperie y a la vez brinda seguridad y comodidad en la espera del transporte público. Su objetivo es facilitar el acceso del usuario al sistema de transporte público, es decir proporcionar facilidad en ingresar y salir del sistema.

Además, las paradas de bus deben complementarse en el diseño y morfología del lugar en relación al contexto urbano, y permitir una fácil identificación tanto para el usuario como para los operadores de bus. Las paradas de autobús son lugares dentro del recorrido de los autobuses de transporte público donde éstos se detienen para permitir el ascenso y descenso de los pasajeros. (Olazabal, 2014)

El transporte público tiene asociados algunos factores cualitativos positivos respecto al vehículo privado que por un lado deberían ayudarle a conseguir recursos y que por el otro deberían ser una parte importante de la motivación de las personas cuando lo utilizan. Estos factores son la cohesión social y territorial, la seguridad, la eficiencia económica (individual y colectiva) y el impacto ambiental. (TOTEM-BUS, 2015)

\section{Especificaciones de diseño y morfología.}

Con respecto al diseño y morfología de los paraderos se consideran algunos aspectos para que su funcionamiento sea eficiente:

- Debe de proporcionar un lugar de refugio, asegurar la protección frente a las condiciones climáticas, especialmente en el invierno. Contar con una cubierta protectora

- El mobiliario empleado en la parada y la infraestructura externa deben ser de fácil mantenimiento y limpieza

- Con respecto al mobiliario, que cuente con suficiente espacio para sentarse, además de tener asientos cómodos.

- Considerar la adecuada ventilación e iluminación en la infraestructura.

- Proporcionar una adecuada identificación e información con respecto a las rutas y paradas en los recorridos de bus.

- Señalética horizontal y vertical clara y de fácil identificación para el usuario y operador de transporte.

- Debe considerarse la facilidad de acceso entre el usuario y el sistema de transporte, además incluir a las personas con capacidades especiales.

El diseño y morfología de las paradas de bus deben ser justificados considerando la demanda de pasajeros, el tiempo de espera, el grado de exposición al viento y al tiempo.(Citada en informe del diagnóstico del Transporte GADM) 


\section{Normas para Establecimiento de Paradas.}

La parada deberá ser eficiente en su funcionamiento, por lo que deberá contar con los siguientes parámetros:

1. La distancia entre paradas deberá ser de 300 metros a 400 metros.

2. Brindar refugio, debe asegurar una protección frente a las condiciones climáticas externas. (Lluvia, viento y sol, extremo)

3. Garantizar una gran calidad en el proyecto y en su ejecución, teniendo en cuenta de forma prioritaria su integración con el entorno, su solidez y durabilidad, así como su fácil limpieza y conservación.

4. Contar con una cubierta protectora y elementos de sustentación, así mismo, siempre que las características del emplazamiento lo permitan, será preciso la instalación de paramentos traseros que cumplan eficazmente con la función protectora ${ }^{i}$ del usuario.

5. Disponer de asientos ergonómicos para los usuarios, los cuales deberán contar con un diseño que impida acostarse en los mismos.

6. Considerar una adecuada higiene, ventilación e iluminación.

7. Deberá contar con un espacio informativo en los paramentos traseros de los paraderos, donde se coloque identificación del nombre de la parada, información de los recorridos de los buses, con señalética visible, y para mejor claridad del aviso, contener mapas de rutas e información municipal, además de ser seguros de fácil acceso tanto para buses como para pasajeros, incluyendo un acceso para personas con capacidades especiales. (Citada en informe del diagnóstico del Transporte GADM)

\section{Señalización horizontal.}

La señalización horizontal está constituida por marcas viales, que son líneas, símbolos y letras que se pintan o se pegan sobre el pavimento, las mismas que informan y orientan a la ciudadanía (conductores, peatones, ciclistas, motociclistas, entre otros), estas señales nos ayudan en la seguridad, eficacia y comodidad de la circulación. (Carlos Kraemer, 2003), citado en informe del diagnóstico del Transporte GADM)

Según la Norma Ecuatoriana Vial 2012, podemos decir que la señalización horizontal se coloca sobre una capa de rodadura de una vía, con respecto a otros tipos de señales, tienen la ventaja de trasmitir su mensaje al conductor en el carril en que circula. (Instituto ecuatoriano de normalización, 2011)

\section{- Parámetros Generales.}

a) Debe ser necesaria.

b) Debe ser visible y llamar la atención.

c) Debe ser legible y fácil de entender.

d) Debe dar tiempo suficiente al usuario para responder adecuadamente.

e) Debe infundir respeto.

f) Debe ser creíble. 


\section{- Diseño Señalización Horizontal.}

a) $\mathrm{Su}$ tamaño, contraste, colores, forma, composición y retrorreflectividad o iluminación, se combinen de tal manera que atraigan la atención de todos los usuarios.

b) Su forma, tamaño, colores y diagramación del mensaje se combinen para que este sea claro, sencillo e inequívoco.

c) Su legibilidad y tamaño correspondan al emplazamiento utilizado, permitiendo en un tiempo adecuado de reacción.

d) Sus características de color y tamaño se aprecien de igual manera durante el día, la noche y periodos de visibilidad limitada.

\section{Clasificación.}

a) Líneas Longitudinales: Se emplean para determinar carriles y calzada; para indicar zonas con o sin prohibición de adelantar; zonas con prohibición de estacionar; y, para carriles de uso exclusivo de determinados tipos de vehículos.

b) Líneas transversales: Se emplean fundamentalmente en cruces para indicar el lugar antes de cual los vehículos deben detenerse y para señalizar sendas destinadas al cruce de peatones o de bicicletas.

c) Simboles y Leyendas: Se emplean tanto para guiar y advertir al usuario como para regular la circulación. Se incluye en este tipo de señalización, flechas, triángulos, ceda el paso, y leyendas tales como pare, bus, carril exclusivo, solo trole, taxis, parada bus, entre otros.

\section{Especificaciones Técnicas.}

Ubicación: La ubicación de la señalización debe ser tal que garantice al usuario que viaja a la velocidad máxima que permite la vía, ver y comprender su mensaje con suficiente tiempo para reaccionar y ejecutar la maniobra adecuada.

Dimensión: Las dimensiones de la señalización dependen de la velocidad máxima de la vía en que se ubican. Están se detallan para cada caso:

- Ojos de Gato, Tachas: Su lado mayor o el diámetro de su base, debe ser de $100 \mathrm{~mm}$ con tolerancia +-5 mm; con altura de 17,5 $\mathrm{mm}$ con tolerancia de $+-2,5 \mathrm{~mm}$.

- Líneas longitudinales: Deben ser de color amarillo, con un ancho mínimo de $100 \mathrm{~mm}$ y máximo $150 \mathrm{~mm}$.

- Línea Segmentada: Para señalizar la separación de carriles de sentido opuesto en una vía de baja velocidad $(<=50 \mathrm{~km} / \mathrm{h}$ ), se debe utilizar una línea de $100 \mathrm{~mm}$ de ancho, con un patrón de 12,00 m y una relación de 3 - 9, es decir 3,00 m pintados y 9,00 m de separación.

- Doble Línea: Dos líneas amarillas paralelas de la misma dimensión 100 mm cada una.

- Línea continua: Son líneas segmentadas que tienen un ancho de $150 \mathrm{~mm}$ a $200 \mathrm{~mm}$, con líneas pintadas de $1,00 \mathrm{~m}$ y espaciamiento de $3,00 \mathrm{~m}$. 
- Línea de Prohibición de estacionamiento: El ancho de esta vía es de $100 \mathrm{~mm}$, se marca a una distancia entre 200 a $800 \mathrm{~mm}$ del bordillo de la calzada dependiendo de la configuración de la vía.

- Línea Ceda el paso: Es una línea segmentada de $600 \mathrm{~mm}$ pintado con espaciamiento de $600 \mathrm{~mm}$, en vías con velocidades de $50 \mathrm{~km} / \mathrm{h}$, el ancho debe ser de $400 \mathrm{~mm}$ y en vías con velocidades superiores el ancho es de $600 \mathrm{~mm}$. (Citado en informe del diagnóstico del Transporte GADM)

La señalización horizontal debe cumplir con los siguientes requisitos mínimos de espesor para su aplicación: zona urbana 300 micras en seco y zona rural 250 micras en seco. (Instituto ecuatoriano de normalización, 2011)

Ancho de carril.

Para el ancho de carril experiencias internacionales demuestran que mayores anchos de carril estimulan velocidades más altas por ello el ancho medido entre centros de líneas se establecen según lo señalado:

Tabla 1: Anchos de carriles

\begin{tabular}{cc}
\hline $\begin{array}{c}\text { Velocidad máxima de la vía } \\
(\mathbf{k m} / \mathbf{h})\end{array}$ & $\begin{array}{c}\text { Ancho del carril } \\
(\mathbf{m})\end{array}$ \\
\hline Menor a 50 (urbana) & Mínimo 3,00 \\
De 50 a 90 (rural) & Entre 3,00 y 3,50 \\
Mayor a 90 (rural) & Entre 3,50 y 3,80
\end{tabular}

Fuente: Instituto ecuatoriano de normalización, 2011

\section{Señalización vertical.}

Según Cal y Mayor r. (2007) y la Norma Ecuatoriana Vial 2012 se puede decir que la señalización vertical sirve para comunicar conductores y peatones de las preferencias que existen entre una vía con otra, estas señales se diseñan de acuerdo con las características técnicas y geométricas de la vía, con el objetivo de brindar información de orden geográfico, turístico, cultural y de servicios.

Estas señales se diseñan de acuerdo con las características de tamaño, contraste, color, composición, retrorreflectividad e iluminación, todas estas características deben estar ajustadas de tal manera que sean captadas fácilmente por los usuarios y así poder realizar las acciones necesarias al momento de percibir el mensaje que transmite la señal.

\section{Clasificación.}

- Señales preventivas: tiene como propósito advertir a los usuarios la existencia de riesgos y/o situaciones especiales presentes en la vía.

- Señales regulatorias: Son tableros con símbolos y/o leyendas, que tienen como función expresar a los usuarios de las vías las prohibiciones y limitaciones sobre su uso. 
- Señales de identificación: Identifica los nombres y nomenclaturas de las calles y las carreteras según su número de ruta y/o kilometraje.

\section{Especificaciones Técnicas.}

- Zona Rural: En vías sin bordillos, la señal debe estar a una distancia libre de por lo menos $600 \mathrm{~mm}$, la separación no debe ser menor de 2,00 m ni mayor de 5,00 m. Debe ser visible bajo iluminación, su altura no debe ser mayor a 1,50 m y en zonas pobladas $2,00 \mathrm{~m}$.

- Zona Urbana: En vías con aceras, las señales deben colocarse, a mínimo $300 \mathrm{~mm}$ del filo del bordillo, la separación mínima debe ser de $500 \mathrm{~mm}$, su altura no debe ser menos de 2,00 m. (Citado en informe del diagnóstico del Transporte GADM)

Para analizar los pavimentos analizando las condiciones de la vía se considera ciertos tipos de falla como se indica en la siguiente tabla:

Tabla 2. Pavimentos flexibles- fallas consideradas

\begin{tabular}{clc}
\hline Falla & \multicolumn{1}{c}{ Tipo-Nombre } & Unidad \\
\hline 1 & Grieta piel de cocodrilo & $\mathrm{m}^{2}$ \\
2 & Exudación de asfalto & $\mathrm{m}^{2}$ \\
3 & Grietas de contracción(bloque) & $\mathrm{m}^{2}$ \\
4 & Elevaciones-hundimiento & $\mathrm{m}^{2}$ \\
5 & Corrugaciones & $\mathrm{m}^{2}$ \\
6 & Depresiones & $\mathrm{m}^{2}$ \\
7 & Grietas de borde & $\mathrm{m}^{2}$ \\
8 & Grietas de reflexión de juntas & $\mathrm{m}^{2}$ \\
9 & Desnivel calzada-hombrillo & $\mathrm{m}^{2}$ \\
10 & Grietas longitudinales y & $\mathrm{m}^{2}$ \\
11 & transversales & $\mathrm{m}^{2}$ \\
12 & Baches y zanjas reparadas & $\mathrm{m}^{2}$ \\
13 & Agregados pulidos & $\mathrm{m}^{2}$ \\
14 & Huecos & $\mathrm{m}^{2}$ \\
15 & Cruce de rieles & $\mathrm{m}^{2}$ \\
16 & Ahuellamiento & $\mathrm{m}^{2}$ \\
17 & Deformación por empuje & $\mathrm{m}^{2}$ \\
18 & Grietas deslizamiento & $\mathrm{m}^{2}$ \\
19 & Hinchamiento & $\mathrm{m}^{2}$ \\
& Disgregación y desintegración & \\
& &
\end{tabular}

Fuente: Método de evaluación de pavimentos PCI (Pavement Condition Index). Cuerpo de Ingenieros de la Armada de E.U.A. Reporte Técnico M-268 (1978) M.Y. Shahin y S.D Khon. Versión en Español por: ING. Augusto Jugo B. Caracas 1987. 


\section{Análisis de las vías de la ciudad de Riobamba.}

Según la información del VII Censo de Población y VI de Vivienda del año 2010, la provincia de Chimborazo cuenta con una población de 458.581 habitantes, de los cuales 187.119 habitantes viven en el área urbana (40.80\%) y 271462 habitantes viven en el área rural (59.20\%). Para efecto de análisis de acuerdo con la proyección de la población, se estima que la infraestructura vial cuenta con una vida útil de 20 años. (Subsecretaría regional 3, 2014). (Citado en informe del diagnóstico del Transporte GADM)

Debido a que, en la rehabilitación de las vías de circulación de la ciudad de Riobamba, se ha realizado obras de infraestructura vial en un:

- $100 \%$, constituido por capa de rodadura con pavimento rígido.

- Parterre central.

- Aceras y bordillos.

- Iluminación y semaforización.

En el componente ambiental se cuenta con arborización en un 100\%; El paso vehicular elevado en las Av. Lizarzaburu y la Av. La Prensa el mismo que cuenta con la infraestructura (Cimentación, Queisson y estribos) y en ejecución obras de superestructura (Encofrado, colocación de hierro para las vigas, etc.), señalización del puente, iluminación y accesos al puente. Drenaje: Implementado 100\%. Pasos peatonales elevados. (Citado en informe del diagnóstico del Transporte GADM).

Se debe tener en consideración las ordenanzas que dictan las autoridades municipales quedando prohibido estacionarse en los carriles destinados al uso exclusivo del transporte público urbano y en las zonas destinadas para estacionamiento y parada de uso exclusivo para el transporte público.

\section{Metodología.}

El principal objetivo de la metodología de la investigación es que las personas estén capacitadas para realizar estudios e investigaciones científicas, en forma lógica y ordenada. Algunos autores proponen como definición que es el estudio sistemático, controlado, reflexivo y crítico de proposiciones hipotéticas sobre las supuestas relaciones que existen entre fenómenos naturales, o que es el proceso sistemático, lógico y organizado para adquirir conocimientos y resolver problemas. ( Salinas,2012)

\section{No Experimental.}

La presente investigación es de tipo no experimental ya realizada en el lugar de los hechos, donde el investigador pudo conocer la realidad a través de la observación mediante el uso de las técnicas e instrumentos necesarios evidenciando el problema de una manera real y verídica.

\section{Tipos de investigación.}




\section{De Campo.}

Se entiende como investigación de campo cuando se presenta mediante la manipulación de una variable externa no comprobada, en condiciones rigurosamente controladas, con el fin de describir de qué modo o porque causas se produce una situación o acontecimiento particular. (Graterol, 2012)

Se realizará este tipo de investigación debido a que el investigador tendrá que estar en las Paradas de bus de transporte público de la ciudad de Riobamba para realizar un análisis sistemático del problema real, con el propósito de describirlo, interpretarlo, entender su naturaleza y sus factores constituyentes, además de explicar las causas y efectos.

\section{Bibliográfico.}

La investigación bibliográfica es la primera etapa del proceso investigativo que proporciona el conocimiento de las investigaciones, de un modo sistemático, a través de una amplia búsqueda de: información, conocimientos y técnicas sobre una cuestión determinada. (Labastida, 1994)

\section{Descripción de las rutas del transporte público en la ciudad de Riobamba.}

Para el transporte público ya sea local o interurbano se obtiene la información origen destino mediante catastros específicos en las terminales correspondientes. La ciudadanía de Riobamba dispone de 16 rutas de bus que realizan recorridos de 06:20 a 21:30, el tipo de recorrido corresponde al circuito cerrado.

Tabla 3. Descripción de rutas línea 01-línea 05

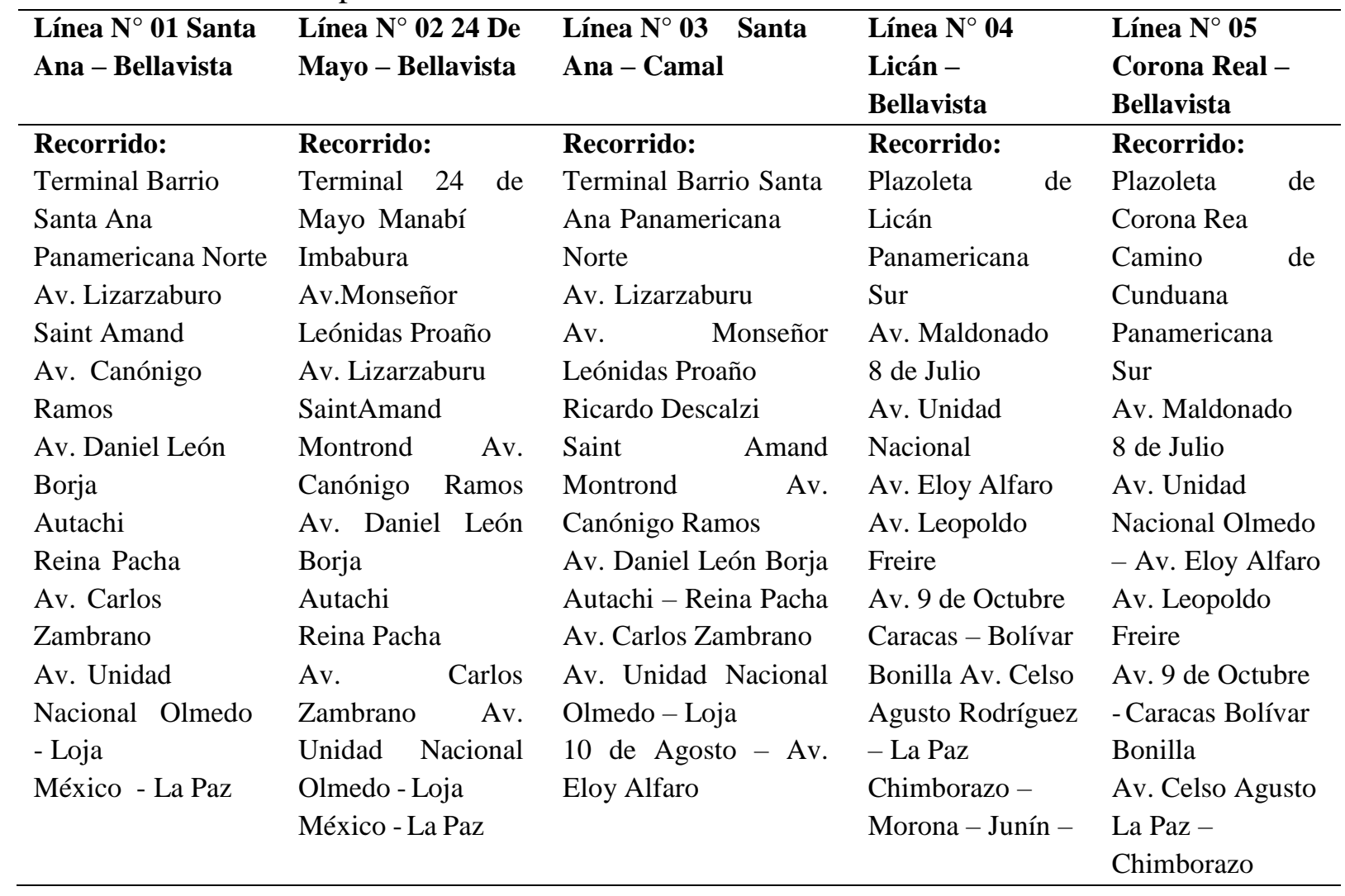




\begin{tabular}{|c|c|c|c|c|}
\hline Av. Celso Agusto & Av. Celso Agusto & Av. Leopoldo Freire & Velasco - Orozco & Morona - Junín - \\
\hline Rodríguez Bolívar & Rodríguez & Quito - Av. Celso & - Carabobo & Velasco - Orozco \\
\hline Bonilla & Bolívar Bonilla & Agusto & Av. Unidad & - Carabobo \\
\hline La Habana & La Habana & Entrada al & Nacional & Av. Unidad \\
\hline Av. 9 de Octubre & Av. 9 de Octubre & Medio Mundo & Av. La Prensa & Nacional \\
\hline Av. Leopoldo & Av. Leopoldo & Barrio El Florecer & Av. Maldonado & Av. La Prensa \\
\hline Freire & Freire Primera & Redondel del mercado & Panamericana Sur & Av. Maldonado \\
\hline Primera & Constituyente & Mayorista & Plazoleta de & Panamericana Sur \\
\hline Constituyente & Diego de Almagro & Av. 9 de Octubre & Licán. & Camino a \\
\hline Diego de Almagro & Orozco - Carabobo & Av. Leopoldo Freire & & Cunduana \\
\hline Orozco & Primera & Av. Eloy Alfaro & & Plazoleta de \\
\hline Carabobo & Constituyente & Guayaquil - Velasco & & Corona Real \\
\hline Primera & Carlos Zambrano & Villarroel- Francia & & \\
\hline Constituyente & Av. Daniel León & Av. Unidad Nacional & & \\
\hline Carlos Zambrano & Borja Av. Canónigo & Carlos Zambrano & & \\
\hline Av. Daniel León & Ramos Saint & Av. Daniel León Borja & & \\
\hline Borja & Amand Montrond & Av. Canónigo Ramos & & \\
\hline Av. Canónigo & Av. Lizarzaburu & Saint $\quad$ Amand & & \\
\hline Ramos & Av. Monseñor & Montrond & & \\
\hline Saint Amand & Leónidas Proaño & Descalzi & & \\
\hline Montrond & Atawascos - Azuay & Av. Lizarzaburu & & \\
\hline Av. Lizarzaburu & Arrawacos - & Panamericana Norte & & \\
\hline Panamericana Norte & Manabí & Terminal Barrio Santa & & \\
\hline Terminal Barrio & Cooperativa de & Ana & & \\
\hline Santa Ana. & vivienda 24 de & & & \\
\hline & Mayo. & & & \\
\hline
\end{tabular}

Fuente: Informe del diagnóstico del Transporte GADM.

Tabla 3. Descripción de rutas línea 06-línea 10

\begin{tabular}{|c|c|c|c|c|}
\hline $\begin{array}{l}\text { Línea } N^{\circ} 06 \\
\text { Miraflores - } \\
\text { Bellavista }\end{array}$ & $\begin{array}{l}\text { Línea } N^{\circ} 07 \\
\text { Inmaculada - El } \\
\text { Rosal }\end{array}$ & $\begin{array}{l}\text { Línea } \mathrm{N}^{\circ} 08 \\
\text { Yaruquies - } \\
\text { Las Abras }\end{array}$ & $\begin{array}{l}\text { Línea } \text { N }^{\circ} 09 \text { Cdla. } \\
\text { Cactus - Licán }\end{array}$ & $\begin{array}{l}\text { Línea } \mathrm{N}^{\circ} 10 \text { San } \\
\text { Antonio - Pinos }\end{array}$ \\
\hline Recorrido: & Recorrido: & Recorrido: & Recorrido: & Recorrido: \\
\hline Plazoleta de Licán & Estacionamiento & Parque de & Estacionamiento: & Barrio San Antonio \\
\hline Panamericana Sur & Barrio la & Yaruquies & Plaza de Licán & del Aeropuert \\
\hline José de Araujo & Inmaculada El troje & Av. Atahualpa & Panamericana Sur Av. & Vía de Ingreso \\
\hline Cooperativa de la & -Vía a Chambo & -Carabobo & Maldonado & Av. José de Sucre \\
\hline Vivienda La Colina & Av. Circunvalación & Carondelet - & Av. 11 de Noviembre & Av. Héroes de Tapi \\
\hline - Esteban Marañón & Av.CelsoA. & Rocafuerte & Av. Canónigo Ramos & Brasil - Gonzalo \\
\hline Juan Machado de & Rodríguez Puruha & Chile - García & Av. De la Prensa- & Dávalos Uruguay - \\
\hline Chávez & - Guayaquil & Moreno Veloz & Autachi Veloz - Colon & Argentinos \\
\hline Juan Bautista & Velasco - & - Espejo & -Barón de Carondelet & 5 de Junio - Veloz \\
\hline Aguirre & Colombia & Av. Cordovéz & - Velasco & Alvarado $-10 \mathrm{de}$ \\
\hline José de Peralta & Francia & Rocafuerte & 12 de Octubre & Agosto Av. Eloy \\
\hline Av. Pedro Vicente & Av. Unidad & Av. 21 de Abril & Alvarado 2 de Agosto & Alfaro \\
\hline Maldonado -8 de & Nacional Av. La & Galo Plaza & -Almagro 24 de Mayo & Celso \\
\hline Julio & Prensa & & - Loja & Rodríguez \\
\hline
\end{tabular}




\begin{tabular}{|c|c|c|c|c|}
\hline Av. Unidad & Av. Canónigo & Jaime Roldós & Chile $-\mathrm{Av}$. & Av. Circunvalación \\
\hline Nacional - Olmedo & Ramos Manuel & Aguilera & Proaño Redondel de & Leopoldo \\
\hline - Av. Eloy Alfaro & Orozco Eduardo & Vicente Ramón & San Luis & Freire Bucarest - \\
\hline Av. Leopoldo & Kingman Joaquín & Roca & Av. Circunvalación & Londres \\
\hline Freire & Pinto & Vía Guano - & Av. Leopoldo Freire & Chile-Valenzuela \\
\hline Av. Edelberto & Manuel Rendón & UNACH Las & Bolívar Bonilla & Boyacá - Espejo \\
\hline Bonilla & Av. Monseñor & Abras & Av. Celso Augusto & Villarroel - \\
\hline Caracas - Bolívar & Leónidas Proaño & Retorno a la & Rodríguez - La Paz & Carabobo Calle 11 \\
\hline Bonilla Av. Celso & Av. Lizarzaburu & UNAC H & Chimborazo - Loja & de Noviembre \\
\hline Augusto Rodríguez & Entrada a FAPANI & Jaime Roldós & Av. Circunvalación & Francia-Colombia \\
\hline La Paz - Pedro & Santa Anita - & Jerónimo & Barrio Perímetro de las & Uruguay \\
\hline Bidón Pineda La & Retorno Santa Anita & Carrión & industrias - Balcón & Av. Unidad \\
\hline trinidad - Morona - & - Entrada a & Av. 21 de Abril & Andino - Los Andes & Nacional \\
\hline Junín Velasco - & FAPANI - Av. & Rocafuerte & Av. Circunvalación & Av. de la Prensa \\
\hline Orozco - Carabobo & Lizarzaburu - Av. & Av. Cordovéz & 5 de Junio - Luz Elisa & Gonzalo Dávalos \\
\hline Av. Unidad & Monseñor Leónidas & 5 de Junio & Borja - Colon - Nueva & Brasil \\
\hline Nacional & Proaño - Manuel & Luz Elisa Borja & York - Uruguay & Av. Héroes de Tapi \\
\hline Av. Pedro Vicente & Rendón-Joaquín & Colon - & Av. Gonzalo Dávalos & Av. Antonio José \\
\hline Maldonado & Pinto Manuel & Villarroel & Barrio Los Pinos & de Sucre Vía de \\
\hline José de Peralta & Orozco-Av. & Pichincha - & Escuela Primera & ingreso a San \\
\hline Juan Bautista & Canónigo Ramos - & Boyacá & Constituyente - Veloz & Antonio del \\
\hline Aguirre & Av. La Prensa & Carabobo & Colegio Riobamba & Aeropuerto Barrio \\
\hline Juan Machado & Av. Manuel Elicio & Av. Atahualpa & Canónigo Ramos - 11 & San Antonio del \\
\hline Chávez Esteban & Flor Veloz - Espejo & GeneralPedro & de Noviembre - Av. & Aeropuerto. \\
\hline Marañón & Argentinos - 5 de & Duchi & Vicente Maldonado - & \\
\hline Cooperativa de la & Junio Veloz - Av. & Fray Astudillo & Ingreso a Licán- & \\
\hline Vivienda La Colina & Celso A. Rodríguez & 24 de Mayo & Estacionamiento Licán. & \\
\hline José de Araujo & Av. Circunvalación & Padre Lobato & & \\
\hline Panamericana Sur & vía a Chambo & Parque de & & \\
\hline Plazoleta de Licán & El troje & Yaruquies. & & \\
\hline
\end{tabular}

Fuente: citado en informe del diagnóstico del Transporte GADM.

Tabla 5. Descripción de rutas línea 11-línea 15

\begin{tabular}{|c|c|c|c|c|}
\hline Línea $\mathbf{N}^{\circ} 11$ & Líinea $\mathrm{N}^{\circ} 12$ San & Línea $\mathrm{N}^{\circ} 13$ Sixto & Línea $\mathrm{N}^{\circ} 14$ & Línea $N^{\circ} 15$ \\
\hline Terminal & Gerardo - El & Duran - 24 De & Libertad - 24 De & Licán-Unach- \\
\hline $\begin{array}{l}\text { Interparroquial - } \\
\text { Mayorista }\end{array}$ & Batan & Mayo & Mayo & Espoch \\
\hline Recorrido: & Recorrido: & Recorrido: & Recorrido: & Recorrido: \\
\hline Estacionamiento Av. & RECORRIDO & Estacionamiento & Parque Central de San & Estacionamiento: \\
\hline Canónigo Ramos & IDA: Plazoleta de & Cdla. Sixto Durán & Luis & Licán (Iglesia) \\
\hline Av. Daniel León & San Gerardo & - Londres Av. & Barrio La Libertad & Av. Pedro \\
\hline Borja & Via Penipe & Juan Félix Proaño & Vía San Luis & Vicente \\
\hline Duchicela & Av. Alfonso & Guayaquil - & Av. Circunvalación - & Maldonado \\
\hline Unidad Nacional & Chávez-Ideal & Velasco & Loja Guayaquil - & Av. La Prensa \\
\hline Chile- Espejo - & Mariana de Jesús & Villarroel - & Velasco Villarroel - & Av. de los Héroes \\
\hline Olmedo & El Espectador & Francia & Francia & Av. Antonio José \\
\hline \multirow[t]{2}{*}{ Eloy Alfaro } & Patria Libre & Av. Unidad & Av. Unidad Nacional & de Sucre Emilio \\
\hline & Mons. Ig. Ordoñez & Nacional & Av. Carlos Zambrano & Estrada \\
\hline
\end{tabular}


ISSN: 2600-5859

\begin{tabular}{|c|c|c|c|c|}
\hline Av. Leopoldo Freire & 5 de Junio - & Av. Carlos & Av. Daniel León & Av. Alfonso \\
\hline Honduras - Costa & Venezuela & Zambrano Av. & Borja Av. La Prensa & Chávez \\
\hline Rica & Carabobo & Daniel León & Av. Maldonado & Av. Edelberto \\
\hline 9 de Octubre & Av. 9 de Octubre & Borja Av. La & Av. Monseñor & Bonilla \\
\hline Leopoldo Freire & Augusto Alzamora & Prensa & Leónidas Proaño & Monseñor José \\
\hline Eloy Alfaro - & San José de Batán & Av. Maldonado & Retorno: Monseñor & Ignacio \\
\hline Guayaquil & San Vicente de & Monseñor & Leónidas Proaño & 5 de Junio - \\
\hline Velasco -Villarroel & Yaruquies & Leónidas Proaño & Av. Maldonado & Orozco \\
\hline Uruguay Av. Unidad & RECORRIDO & Retorno: & Av. La Prensa & Av. Miguel Ángel \\
\hline Nacional & VUELTA: San & Monseñor & Av. Daniel León & León Veloz - \\
\hline Av. La Prensa & Vicente de & Leónidas Proaño & Borja Autachi - & Av. La Prensa \\
\hline Av. Canónigo Ramos & Yaruquies & Av. Maldonado & Reina Pacha Av. & Av. Pedro \\
\hline Terminal & San José de Batan & Av. La Prensa & Carlos Zambrano & Vicente \\
\hline Intraparroquial. & Augusto Alzamora & Av. Daniel León & Av. Unidad Nacional & Maldonado \\
\hline & Av. 9 de Octubre & Borja Autachi - & Olmedo - Loja & Licán (Iglesia) \\
\hline & Carabobo -12 de & Reina Pacha Av. & 10 de Agosto & \\
\hline & Octubre - & Carlos Zambrano & Av. Eloy Alfaro - & \\
\hline & Rocafuerte & Av. Unidad & Olmedo Av. Félix & \\
\hline & Ayacucho - Tarqui & Nacional & Proaño & \\
\hline & Mons. Jacinto & Olmedo - Loja & Cdla. Primera & \\
\hline & Ordoñez Patria & 10 de Agosto & Constituyente & \\
\hline & Libre - El & Av. Eloy Alfaro & Av. Circunvalación & \\
\hline & Espectador - & Olmedo - Av. & Vía San Luis & \\
\hline & Mariana de Jesús - & Félix Proaño - & Barrio La Libertad & \\
\hline & Ideal & Londres & Parque Central de San & \\
\hline & Av. Alfonso & Estacionamiento & Luis & \\
\hline & Chávez Vía a & Cdla. Sixto & & \\
\hline & Penipe & Durán. & & \\
\hline & Plazoleta de San a & & & \\
\hline & Gerardo & & & \\
\hline
\end{tabular}

Fuente: citado en informe del diagnóstico del Transporte GADM.

Tabla 6. Descripción de rutas línea 16

\begin{tabular}{l}
\hline \multicolumn{1}{c}{ LÍNEA $\mathbf{N}^{\circ}$ 16 CALPI - LA PAZ } \\
\hline Recorrido: \\
Estacionamiento de Calpi Panamericana Sur \\
Av. Pedro Maldonado \\
Av. Saint Ammand \\
Av. Canónigo Ramos \\
Av. La Prensa \\
Manuel Elisio Flor \\
Veloz - España - Carondelet Velasco - 2 de Agosto \\
Diego de Almagro - Orozco \\
Av. Miguel Ángel León - Veloz Av. La Prensa - Av. Canónigo \\
Ramos \\
Av. Saint Ammand \\
Av. Maldonado \\
Panamericana Sur Estacionamiento de Calpi.
\end{tabular}

Fuente: Informe del diagnóstico del Transporte GADM. 


\section{Análisis de resultados.}

$\mathrm{Al}$ analizar las características geométricas del ancho de carril y de la infraestructura de paradas de bus de los corredores de transporte público de la ciudad de Riobamba, divididos en 10 grupos abarcan a las 16 líneas de estudio, estos grupos se realizan puesto que existen líneas con rutas parecidas se diferencian en las terminales como puntos de inicio y llegada, como se puede observar en las tablas 1,2,3 y 4 .

- Grupo 1: línea 1,2,3

- Grupo 2: línea 4,5,6

- Grupo 3: línea 7,11

- Grupo 4: línea 8

- Grupo 5: línea 9

- Grupo 6: línea 10

- Grupo 7: línea 12

- Grupo 8: línea 13,14

- Grupo 9: línea 15

- Grupo 10: línea 16

En las siguientes figuras se observan los resultados y criterios de evaluación de cada uno de los grupos para: tipo de señalización, estructura de parada y ancho de carril. La figura 1 indica el Tipo de Señalización, existe una mayor presencia de señalización vertical en las rutas de transporte público de la ciudad de Riobamba con un $65 \%$ y un $56 \%$ de existencia de señalización horizontal.

Figura 1: Tipo de señalización

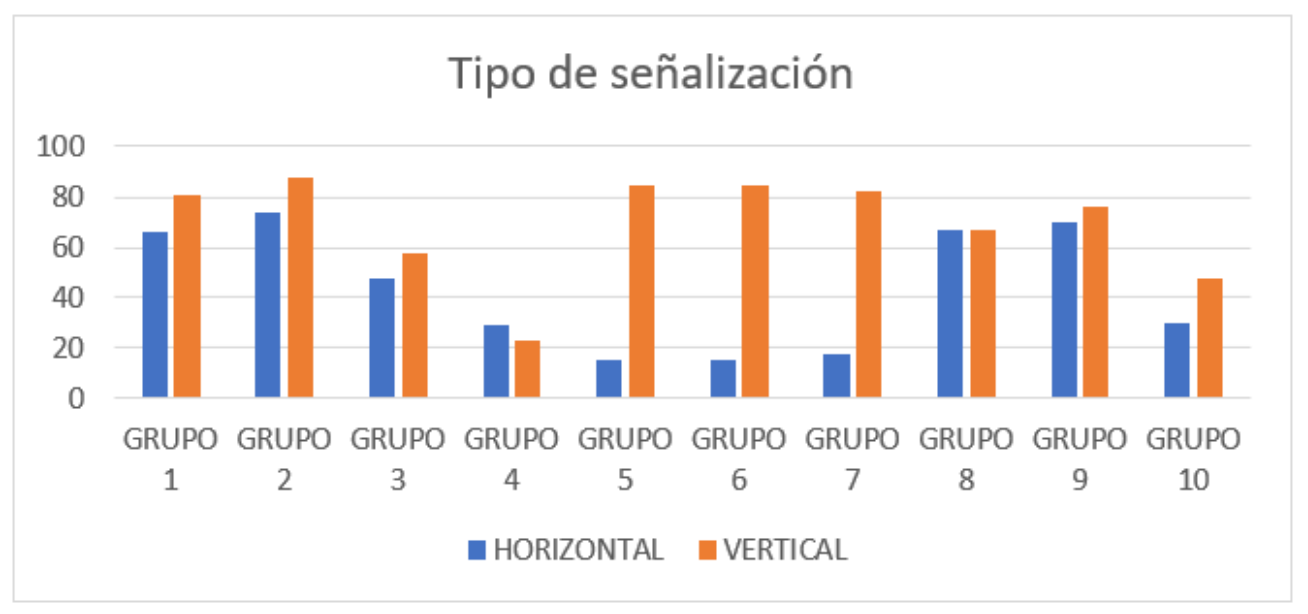

Elaborado: Grupo de investigación.

Fuente: Informe del diagnóstico del Transporte GADM. 
La figura 2 indica que un $70 \%$ no cuenta con una infraestructura de estructura de parada y el $30 \%$ cuenta con una estructura de paradas en las rutas de transporte público.

Figura 2. Estructura de parada

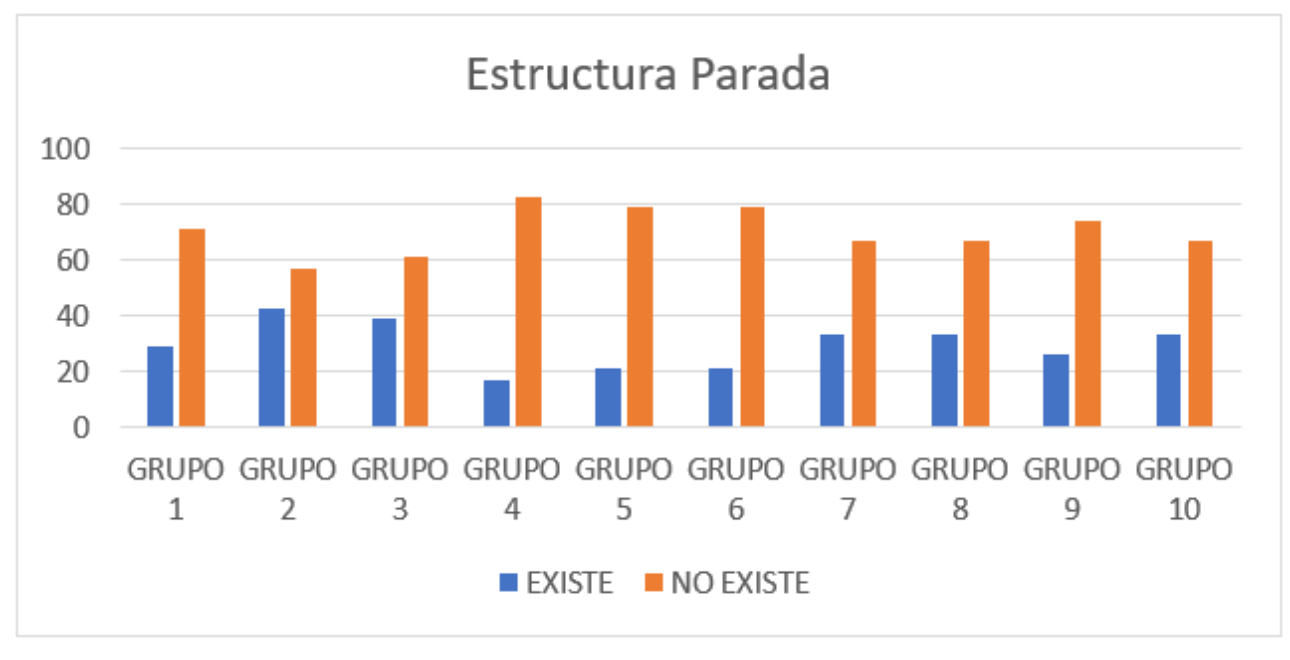

Elaborado: Grupo de investigación.

Fuente: Informe del diagnóstico del Transporte GADM.

La figura 4 y 5 indican las condiciones del ancho de carril bajo un análisis cuantitativo y cualitativo se evidencia que existe un cumplimiento normativo en el ancho de carril establecido en las rutas de transporte público, se puede observar los grupo 4 y 10 tienen condiciones deficientes.

Figura 3: Ancho de carril análisis cuantitativo

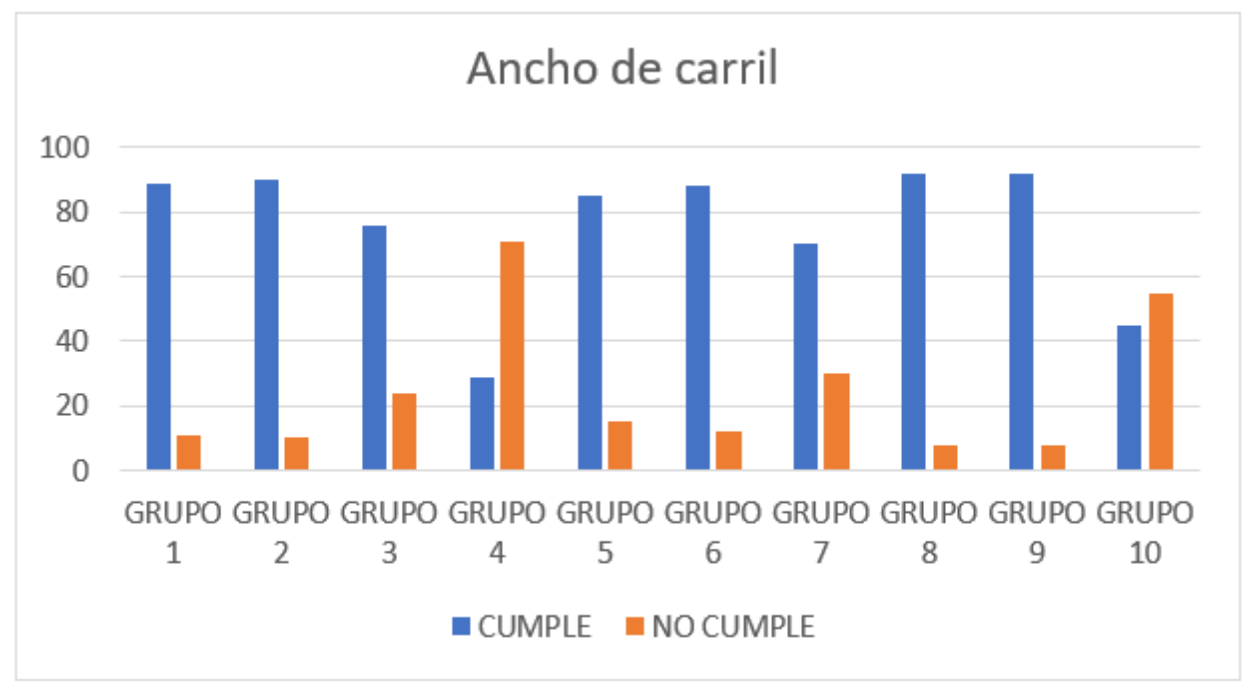

Elaborado: Grupo de investigación.

Fuente: Informe del diagnóstico del Transporte GADM. 
Figura 4. Ancho de carril análisis cualitativo

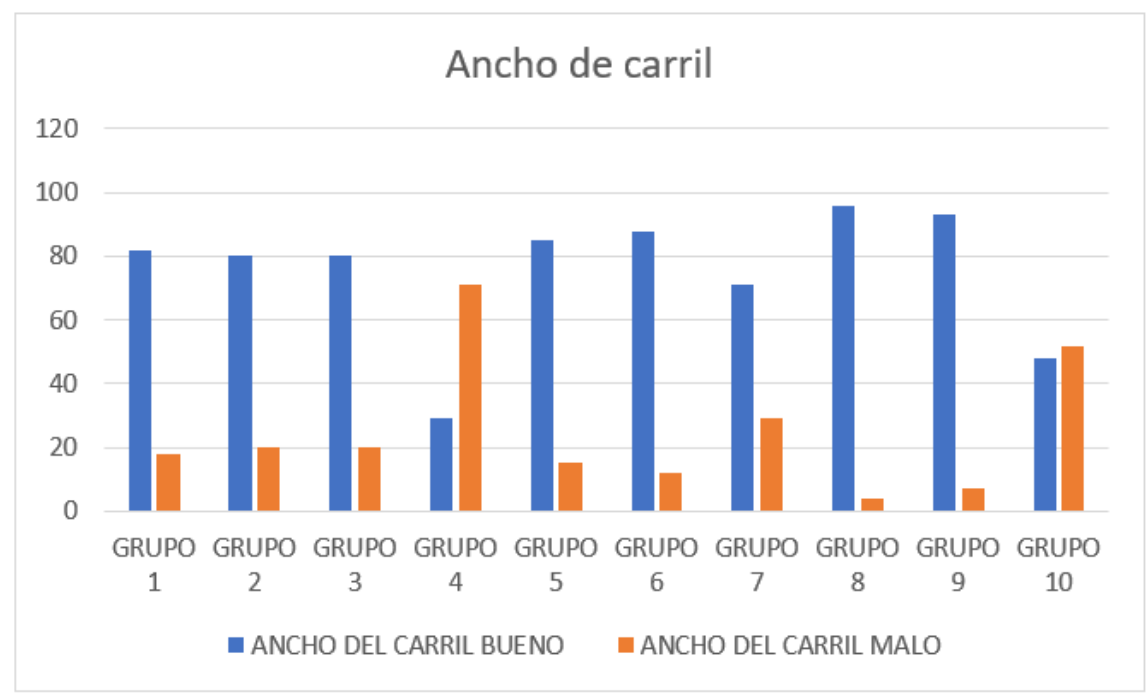

Elaborado: Grupo de investigación.

Fuente: Informe del diagnóstico del Transporte GADM.

\section{Conclusiones:}

- Actualmente la señalización vertical y horizontal necesita un mejoramiento oportuno para brindar mayor eficiencia a los usuarios que utilizan el transporte público como medio primordial para su movilización.

- La señalización horizontal se ve afectada por el índice de ausentismo en las vías de la ciudad.

- La necesidad se prioriza como medida de acción para contrarrestar esta deficiencia que conlleva al deterioro de las condiciones actuales de infraestructura y así como también a la problemática de accidentes de tránsito que se mantienen en la ciudad.

- El ancho de carril necesita ser analizado en las rutas delas líneas del grupo 4 y 10 debido a que las condiciones geométricas no son óptimas para la circulación de las unidades de transporte público.

\section{Referencias bibliográficas:}

Carlos Kraemer, J. M. (2003). Ingenieria de Carreteras. Madrid - España : Concepcion Fernandez Madrid.

Subsecretaría regional 3. (2014). Resumen Ejecutivo De Viabilidad De Proyectos. riobamba .

Totem-Bus. (02 de Abril de 2015). Definicion, parametros, tipologia, calidad del diseño y materiales. Recuperado el 05 de Julio de 2017, de Definicion, parametros, tipologia, calidad del diseño y materiales.: https://www.google.com/search?ei=9p_fWr8HsGxgge- 
irnoBA\&q=Definici\%C3\%B3n+de+parada+de+autob\%C3\%BAs+especificaciones $+\mathrm{d}$ e+dise $\%$ C3\%B1o\&oq=Definici\%C3\%B3n+de+parada+de+autob\%C3\%BAs+especifi caciones+de+dise\%C3\%B1o\&gs_l=psy-ab.3..33i160k1.18660.24922.0.25660.28

Salinas, P. (2012). Metodología de la investigación. Merida: Universidad de Los Andes.

Ministerio de Transporte y Obras Públicas del Ecuador. (2013). Libro a normas para estudios y diseños viales.

Ministerio del Interior. (2015). Normas y señales reguladoras de la circulación. Madrid: Nipo.pag. 215

Olazabal, Nerea ""Un nuevo concepto de parada de autobús urbano como una combinación de nuevos servicios y requerimientos de los usuarios"".Leslabay, Marcelo y Morer, Paz. Proyecto fin de grado. Universidad de Navarra, 2014

Instituto ecuatoriano de normalización. (2011) .Reglamento técnico ecuatoriano. QuitoEcuador.

Método De Evaluación De Pavimentos Pci (Pavement Condition Index). Cuerpo de Ingenieros de la Armada de E.U.A. Reporte Técnico M-268 (1978) M.Y. Shahin y S.D Khon. Versión en Español por: ING. AUGUSTO JUGO B. Caracas 1987.

Martinez Filho, A., Vasconcellos, E. A., \& Paulino, H. (2014). Gestión del tránsito. Serie de cuadernos de investigación del Observatorio de Movilidad Urbana de América Latina y el Caribe;2/2014, Buenos Aires: CAF. Retrieved from http://scioteca.caf.com/handle/123456789/792. 


\section{PARA CITAR EL ARTÍCULO INDEXADO.}

Velasco Castelo, G. M., Guerrero Godoy, A. P., \& Aguilar Miranda, G. J. (2020). Análisis de las características geométricas del ancho de carril e infraestructura en las paradas de bus del transporte público urbano en la ciudad de Riobamba. ConcienciaDigital, 3(3), 350-366. https://doi.org/10.33262/concienciadigital.v3i3.1325

\section{LCiencia}

El artículo que se publica es de exclusiva responsabilidad de los autores y no necesariamente reflejan el pensamiento de la Revista Conciencia Digital.

El artículo queda en propiedad de la revista y, por tanto, su publicación parcial y/o total en otro medio tiene que ser autorizado por el director de la Revista Conciencia Digital.

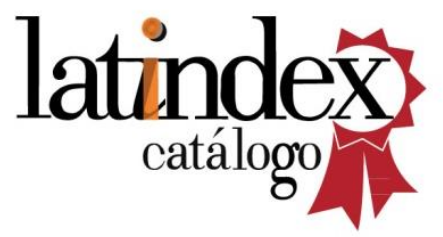

